\title{
THE RIESZ DECOMPOSITION FOR VECTOR-VALUED AMARTS
}

\author{
BY G. A. EDGAR AND L. SUCHESTON 1
}

Communicated by Alexandra Bellow, April 12, 1976

Let $(\Omega, F, P)$ be a probability space, $\mathbf{N}=\{1,2, \ldots\}$, and let $\left(F_{n}\right)_{n \in \mathbf{N}}$ be an increasing sequence of $\sigma$-algebras contained in $F$. A stopping time is a mapping $\tau: \Omega \rightarrow \mathbf{N} \cup\{\infty\}$, such that $\{\tau=n\} \in F_{n}$ for all $n \in \mathbf{N}$. The collection of bounded stopping times is denoted by $T$; under the natural ordering $T$ is a directed set 'filtering to the right'.

Let $\mathbf{E}$ be a Banach space and consider a sequence $\left(X_{n}\right)_{n \in \mathrm{N}}$ of E-valued random variables adapted to $\left(F_{n}\right)$, i.e., such that $X_{n}: \Omega \rightarrow \mathbf{E}$ is $F_{n}$-strongly measurable. $E X$ (expectation of $X$ ) is the Pettis integral of $X ; E_{A} X$ denotes $E\left(1_{A} \cdot X\right)$. The sequence $\left(X_{n}\right)$ is called an amart iff each $X_{n}$ is Pettis integrable and $\lim _{T} E\left(X_{\tau}\right)$ exists in the strong topology of $\mathbf{E}$.

The real Riesz decomposition theorem for amarts [4] asserts that an amart $X_{n}$ can be uniquely written as a sum of a martingale $Y_{n}$, and an amart $Z_{n}$ that converges to zero in nearly all possible ways: $Z_{n} \rightarrow 0$ a.e. and in $L^{1}$, and $Z_{\tau} \rightarrow$ 0 in $L^{1}$.

As a consequence of this result, and of the real amart convergence theorem [1] - the first important result involving discrete parameter amarts-we obtain

THEOREM 1. Let $\mathbf{E}=\mathbf{R}$. If $\left(X_{n}, F_{n}\right)$ is an amart, then (and only then) for each increasing sequence $\tau_{n} \geqslant n$ in $T, E^{F^{n}} X_{\tau_{n}}-X_{n} \rightarrow 0$ a.e. and in $L^{1}$.

The Banach-valued Riesz decomposition is the main result of the present note. The Pettis norm of a random variable $X$ is $\|X\|=\sup E|f(X)|$ where the supremum is over all $f \in \mathbf{E}^{\prime}$ with $|f| \leqslant 1$ [6].

A potential is an amart that converges to zero in the Pettis norm. A sequence of adapted random variables is said to be of class (B) iff $\sup _{T} E\left|X_{\tau}\right|<\infty$. We prove

TheOREM 2 (RIESz DeCOMPOSITION). Let $\mathbf{E}$ be a Banach space with the Radon-Nikodym property and let $\left(X_{n}, F_{n}\right)$ be an E-valued amart such that

$$
\lim \inf E\left|X_{n}\right|<\infty \text {. }
$$

(i) $X_{n}$ can be uniquely written as the sum of a martingale $Y_{n}$ and a

AMS (MOS) subject classifications (1970). Primary 60G40, 60G45.

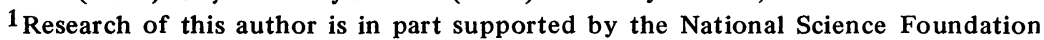
grant MPS 72-04752 A03. 
potential $Z_{n} .\left(Z_{\tau}\right)_{\tau \in T}$ converges to zero in Pettis norm.

(ii) If $\mathbf{E}^{\prime}$ is separable and $\left(X_{n}, F_{n}\right)$ is of class (B), then $Z_{n} \rightarrow 0$ a.e. weakly.

SKETCH OF PROOF. (Complete proof will appear elsewhere). (i) For each $A \in F_{m}, \lim E_{A} X_{n}=\mu_{m}(A)$ exists (cf. [3]). (1) implies that $\mu_{m}$ has finite variation. $Y_{m}=d \mu_{m} / d P$ is a martingale and $Z_{m}=X_{m}-Y_{m}$ satisfies $E_{A} Z_{m}$ $\longrightarrow 0 \forall A \in F_{m}$. Let $\epsilon_{i} \downarrow 0$. For each $m$ choose $A_{m} \in F_{m}$ so that

$$
\sup _{A \in F_{m}}\left|E_{A} Z_{m}\right|-\left|E_{A_{m}} Z_{m}\right|<\epsilon_{m} .
$$

We can find an integer $n_{m}>m$ such that $\left|E_{A_{m}^{c}}^{c} Z_{n_{m}}\right|<\epsilon_{m}$. Define a stopping time $\tau_{m}$ by $\tau_{m}=m$ on $A_{m} ; \tau_{m}=n_{m}$ on $A_{m}^{c}$. Then for each $m, \mid E_{A_{m}} Z_{m}-$ $E Z_{\tau_{m}}|=| E_{A_{m}^{c}}^{c} Z_{n_{m}} \mid<\epsilon_{m}$. Since $Z_{n}$ is an amart, $\lim _{m} E Z_{\tau_{m}}=\lim _{m} E Z_{m}=0$. It follows that $E_{A_{m}} Z_{m} \rightarrow 0$; hence, by (2), $\sup _{A \in F_{m}}\left|E_{A} Z_{m}\right| \longrightarrow 0$ which implies that $\left\|Z_{m}\right\| \rightarrow 0$.

For each increasing sequence of bounded stopping times $\tau_{n},\left(Z_{\tau_{n}}\right)_{n \in \mathrm{N}}$ is an amart with respect to $\left(F_{\tau_{m}}\right)_{m \in \mathbf{N}}[4]$. Therefore $\left\|Z_{\tau_{m}}\right\| \rightarrow 0$; it follows that $\lim _{T}\left\|Z_{\tau}\right\|=0$.

The proof of (ii) uses the vector amart convergence theorem [3] .

In the discussing of examples relevant to Theorem 2, the following result is useful: For any E, any amart, the Riesz decomposition holds if and only if $d \mu_{m} / d P$ exists for each $m$.

EXAMPLE 1. The assumption that $\mathbf{E}$ has the Radon-Nikodym property cannot be omitted. Let $\left\{e_{n}^{i}, n \in \mathbf{N}, 1 \leqslant i \leqslant 2^{n}\right\}$ be the standard basis for the Banach space $c_{0}$ (in any order). Let $A_{n}^{i} \cap A_{n}^{j}=\varnothing$ if $i \neq j$, and $P\left(A_{n}^{i}\right)=2^{-n}$. Let

$$
X_{n}=\sum_{k=1}^{n} \sum_{i=1}^{2^{k}} e_{k}^{i} 1_{A_{k}^{i}} .
$$

Let $F_{n}=F, n=1,2, \ldots\left(X_{n}, F_{n}\right)$ is a bounded amart (cf. [3]), but the Riesz decomposition fails.

EXAMPLE 2. Assumption (1) cannot be omitted. Let $\left(e_{i}\right)$ be the usual basis of $\mathbf{E}=l_{p}$ for some $p, 1 \leqslant p<2$. Let $Z_{n}$ be independent real random variables with $P\left(Z_{n}=1\right)=P\left(Z_{n}=-1\right)=1 / 2$. Set $X_{n}=\Sigma_{k=1}^{n} a_{k} e_{k} Z_{k}$ where $a_{k}$ are positive constants with $\Sigma_{k} a_{k}^{p}=\infty, \Sigma a_{k}^{2}<\infty$. Then $\lim E\left|X_{n}\right|=\infty$, and one proves that $X_{n}$ is an amart with respect to the constant sequence of $\sigma$-algebras $F$, that does not have the Riesz decomposition.

The first example in [3] (or [4]) shows that a bounded potential in a Hilbert space need not converge a.e. strongly, or in $L_{E}^{1}$ norm; the second example shows that an $L^{1}$ bounded potential not of class (B) need not converge a.e. weakly. 


\section{REFERENCES}

1. D. G. Austin, G. A. Edgar and A. Ionescu Tulcea, Pointwise convergence in terms of expectations, Z. Wahrscheinlichkeitstheorie und Verw. Gebiete 30 (1974), 17-26. MR 50 \#11402.

2. A. Bellow, On vector-valued asymptotic martingales, Proc. Nat. Acad. Sci. U.S.A. (to appear).

3. R. V. Chacon and L. Sucheston, On convergence of vector-valued asymptotic martingales, Z. Wahrscheinlichkeitstheorie und Verw. Gebiete 33 (1975), 55-59.

4. G. A. Edgar and L. Sucheston, Amarts: A class of asymptotic martingales, J. Multivariate Analysis (to appear).

5. - Les amarts: une classe de martingales asymptotiques, C. R. Acad. Sci. Paris (to appear).

6. B. J. Pettis, On integration in vector spaces, Trans. Amer. Math. Soc. 44 (1938), 277-304.

DEPARTMENT OF MATHEMATICS, OHIO STATE UNIVERSITY, COLUMBUS, OHIO 43210 